\title{
Cellular players of hematopoietic stem cell mobilization in the bone marrow niche
}

\author{
Joshua Tay $^{1} \cdot$ Jean-Pierre Levesque $^{2} \cdot$ Ingrid G. Winkler $^{1}$
}

Received: 16 November 2016 / Revised: 21 November 2016 / Accepted: 29 November 2016 / Published online: 10 December 2016

(c) The Japanese Society of Hematology 2016

\begin{abstract}
Hematopoietic stem cells (HSC) reside in perivascular regions of the bone marrow (BM) embedded within a complex regulatory unit called the niche. Cellular components of HSC niches include vascular endothelial cells, mesenchymal stromal progenitor cells and a variety of mature hematopoietic cells such as macrophages, neutrophils, and megakaryocytes - further regulated by sympathetic nerves and complement components as described in this review. Three decades ago the discovery that cytokines induce a large number of HSC to mobilize from the BM into the blood where they are easily harvested, revolutionised the field of HSC transplantation-curative for immune-deficiencies and some malignancies. However, despite now routine use of granulocyte-colony stimulating factor (G-CSF) to mobilise HSC for transplant, only in last 15 years has research on the mechanisms behind why and how HSC can be induced to move into the blood began. These studies have revealed the complexity of the niche
\end{abstract}

J.-P. Levesque and I. G. Winkler are co-senior authors and co-corresponding authors.

Jean-Pierre Levesque

jp.levesque@mater.uq.edu.au

$\triangle$ Ingrid G. Winkler

Ingrid.winkler@mater.uq.edu.au

1 Stem Cells and Cancer Group, Blood and Bone Diseases Program, Faculty of Medicine and Biomedical Sciences, Mater Research Institute, Translational Research Institute, University of Queensland, 37 Kent Street, Woolloongabba, QLD 4102, Australia

2 Stem Cell Biology Group, Blood and Bone Diseases Program, Faculty of Medicine and Biomedical Sciences, Mater Research Institute, Translational Research Institute, University of Queensland, 37 Kent Street, Woolloongabba, QLD 4102, Australia that retains HSC in the BM. This review describes how BM niches and HSC themselves change during administration of G-CSF-or in the recovery phase of chemotherapy-to facilitate movement of HSC into the blood, and research now leading to development of novel therapeutics to further boost HSC mobilization and transplant success.

Keywords Hematopoietic stem cells $\cdot$ Stem cell niche Mobilization $\cdot$ Bone marrow

\section{Introduction}

Hematopoietic stem cells (HSC) are rare cells residing in the bone marrow (BM). Their role is to generate the hematopoietic progenitor cells (HPC) needed to replenish the blood and immune system.

The discovery three decades ago that administration of the cytokine granulocyte colony-stimulating factor (G-CSF), or the recovery phase from chemotherapy, triggered the movement of HSCs from the BM into the peripheral blood (mobilization) from where they can be harvested, has revolutionized HSC transplant (formerly known as BM HSC transplant). HSC transplantation is curative and nowadays part of the standard of care for $\sim 70$ diseases including inherited immunodeficiencies, BM failure, myelodysplasia and many relapsed hematopoietic malignancies. Mobilized blood is now the main source of HSC for transplantation, almost completely replacing $\mathrm{BM}$ aspiration, with the advantage of being easier and less painful for donors and a more rapid reconstitution in transplant recipients. Over one million transplantations have been performed with mobilized blood as a source of HSC. Notably, the use of mobilized blood HSC to transplant patients began decades before any understanding of 


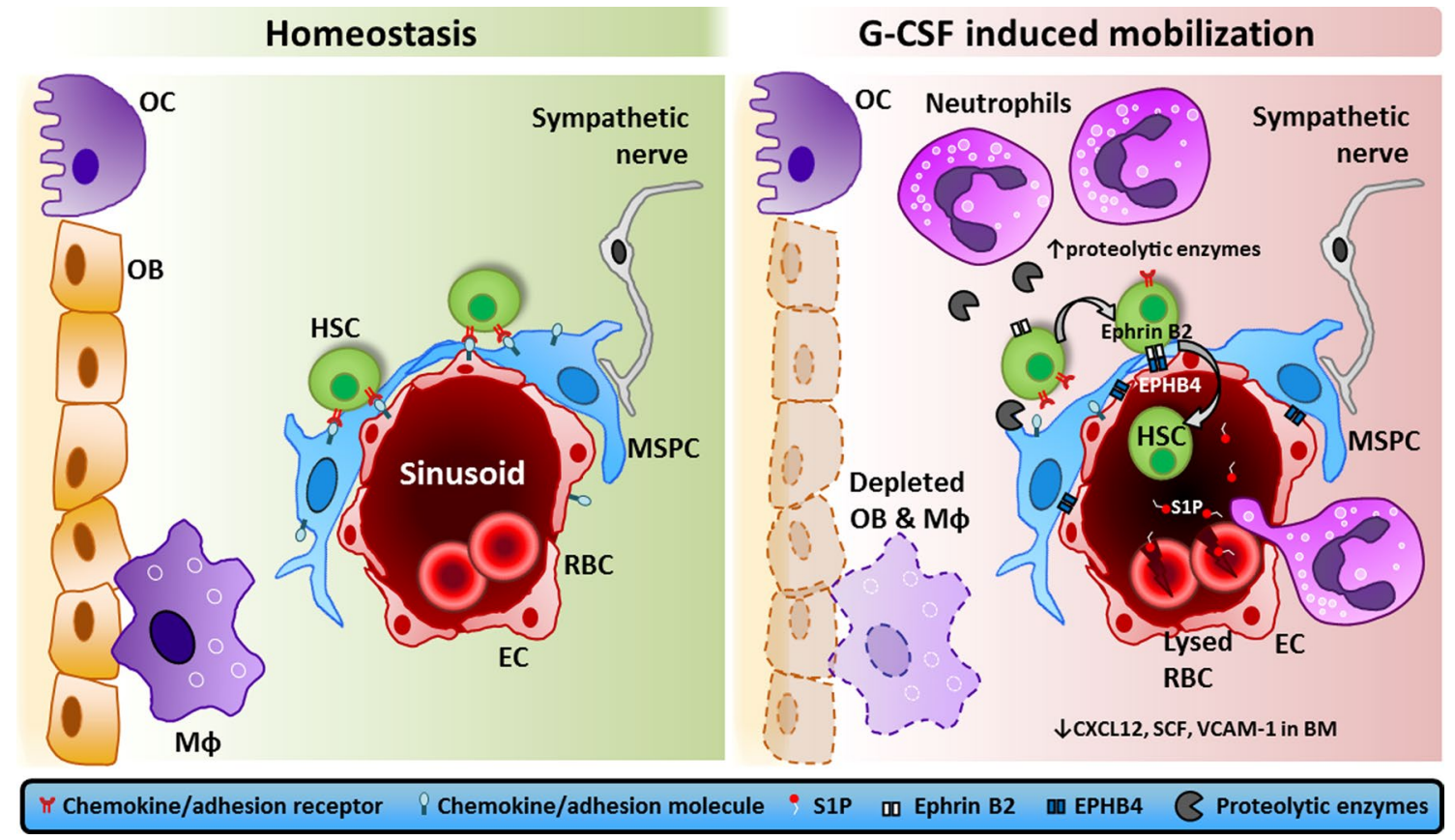

Fig. 1 Overview of the effects of G-CSF on key cellular components in the BM niche. During homeostasis, HSCs are retained in the BM niche via chemokine/adhesion molecules expressed primarily by mesenchymal stromal progenitor cells (MSPCs) and endothelial cells (EC). G-CSF expands neutrophil numbers in the BM, which releases proteolytic enzymes that cleave and inactivate chemokine/adhesion factors such as CXCL12, SCF, and VCAM-1. Macrophages $(\mathrm{M} \varphi)$ are depleted following G-CSF administration, which is associated with osteoblast (OB) ablation and reduced CXCL12, SCF, and VCAM-1

the mechanisms - why HSC reside in the BM and what makes them move into the blood following G-CSF or during recovery from chemotherapy. These mechanistic studies have now unravelled the complexity of the BM niches, their cellular and molecular composition, and how they dynamically interact with HSC in response to stressors in the body. The picture emerging is of a complex system comprising a HSC embedded within a regulatory niche composed of endothelial cells (EC), mesenchymal stromal progenitor cells (MSPC) and critical involvement of mature hematopoietic neutrophils and macrophages with further regulation by sympathetic nervous and complement systems. The role each of these cellular players of the HSC niche play in contributing to mobilization is reviewed herein. Importantly although many agents or physiological events (such as vigorous exercise) can 'mobilize' a small proportion of HSC or HPC to levels a few fold above baseline, the therapeutic regimes are able to mobilize large numbers of HSC to levels at least 25- to 100-fold above baseline (median $\sim 140 \times 10^{6}$ circulating $\mathrm{CD} 34^{+}$cells per litre blood after 4-6 days of G-CSF) [1]. In the former instance, demargination of HSC from BM perivascular niches may be the more appropriate term in comparison to expression in the $\mathrm{BM}$, which is partially explained by sympathetic nervous downregulation of MSPC expression of CXCL12, SCF, and VCAM-1. G-CSF also induces EPHB4 clustering on EC away from the lumen of the sinusoid, which couples to Ephrin B2 on HSC and promotes transendothelial migration. G-CSF activates the complement system, leading to hemolysis and the release of chemotactic sphingosine-1-phosphate (S1P) in the blood. These interconnected mechanisms lead to HSC mobilization into the blood

the pronounced mobilization that occurs following G-CSF where parabiotic mice studies have suggested that around $20 \%$ of total BM reconstituting HSC entered the blood and relocated in response to G-CSF [2].

In this review we will summarize the different cellular components of the BM that participate in the therapeutic mobilization of HSC from their niche reservoir into the peripheral blood as observed in response to G-CSF and cytotoxic agents such as cyclophosphamide (CYP). As reviewed and detailed elsewhere [3-6], these mechanisms of HSC mobilization involve major changes in the HSC niches, particularly in respect to molecules that actively retain $\mathrm{HSC}$ within their niches, such as vascular cell adhesion molecule-1 (VCAM-1), transmembrane kit ligand (or stem cell factor-SCF), the chemokine CXCL12, and its receptor CXCR4. HSC mobilization in response to cytokines such as G-CSF or to CYP involve several mechanisms working in concert as detailed in this review, such as proteolytic cleavage and down-regulation of expression of key retention factors, all of which involve several interconnected cellular mechanisms mediated by BM myeloid cells, macrophages, MSPCs, ECs, adrenergic nerves and erythrocytes. These roles are summarised in Fig. 1 and Table 1. 


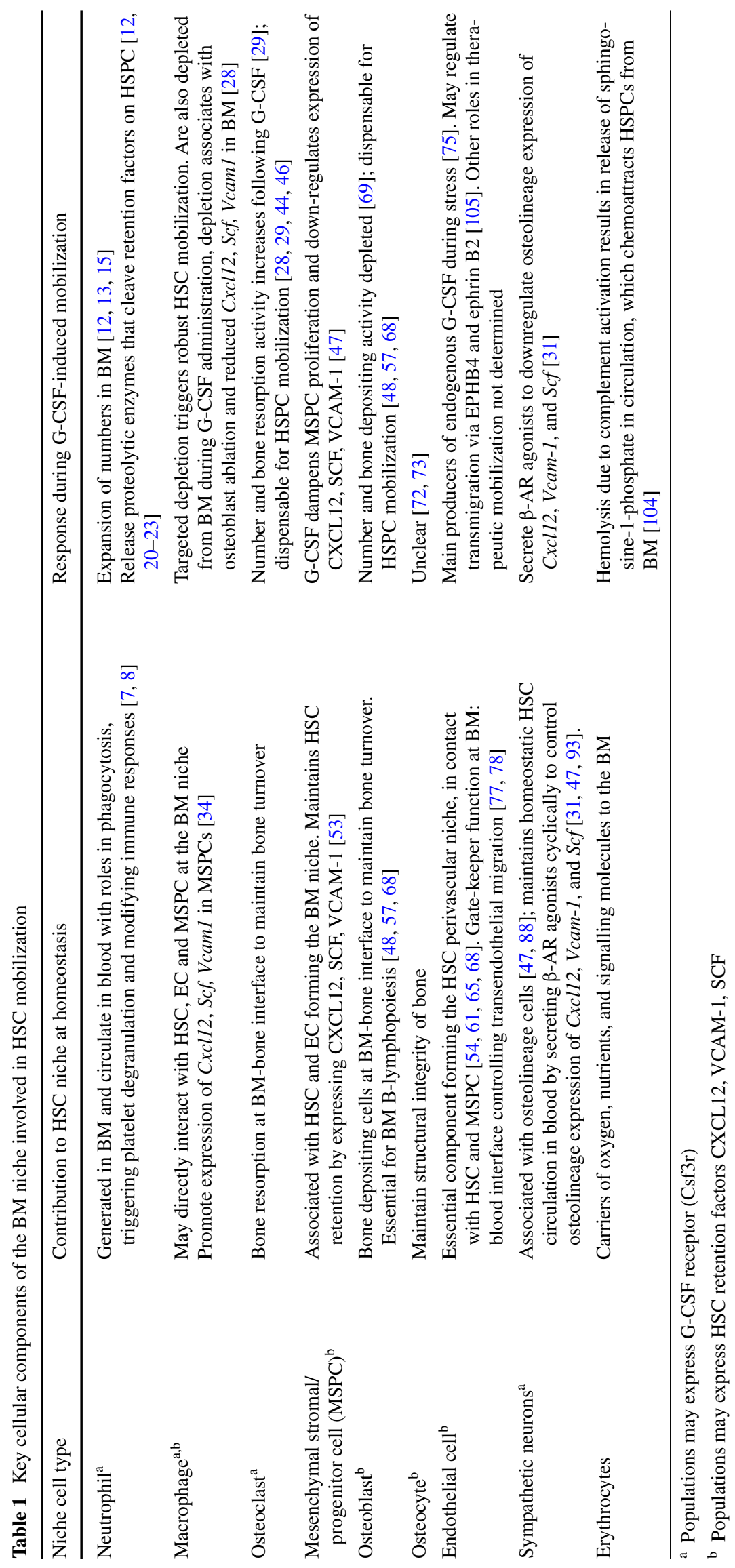




\section{Myeloid cells within the BM}

\section{Neutrophils}

Neutrophils are well known for their rapid response to inflammatory signals by migrating to sites of infections and inflammation, degranulating their content to kill microbes and phagocytosis, with release of cytokines by neutrophils also contributing to the recruitment of other immune leukocytes (reviewed in [7, 8]). In steady state, about a third of all circulating senescent neutrophils are phagocytosed by in the BM by $\mathrm{CD} 169^{+}$resident macrophages, which subsequently release G-CSF to promote granulopoiesis [9, 10], and mobilization of HPCs via activation of cholesterolsensing nuclear receptors [11].

In the context of pharmacological mobilization, neutrophils are activated and expand their pool size significantly in the BM in response to mobilizing agents G-CSF, cyclophosphamide (CYP), IL-8, and GRO $\beta$ [12-15]. Several studies over the decades have demonstrated that neutrophil depletion such as induced by anti-Gr-1 antibody administration or by homozygous deletion of the $C s f 3 r$ gene encoding the G-CSF receptor, severely hampered HSPC mobilization in response to G-CSF, cyclophosphamide, IL-8 or GRO $\beta$ [14, 16-18]. As the main biological response to G-CSF administration in vivo is a pronounced neutrophilia, while anti-Gr1 administration or $C s f 3 r$ gene deletion leads to a profound neutropenia, this led to the view that neutrophils play an important role in HSPC mobilization in response to these agents [19].

Initial studies performed in BALB/c and $129 \mathrm{SvJ}$ strains, and evidence from human samples showed that following G-CSF or cyclophosphamide, BM neutrophils release proteases such as neutrophil elastase, cathepsin G, matrix metalloproteinase (MMP)-9, and dipeptidyl peptidase I (required for functional activation of a wide variety of serine proteases) $[12,20,21]$. These proteases then cleave and inactivate cell adhesion molecules such as VCAM-1 and Kit ligand in the BM as well as the chemokine CXCL12 [12, 20-23]. Active proteases are inhibited by specific inhibitors such as serine-protease inhibitors (serpins) and tissue inhibitors of metalloproteinases (TIMPs) in many tissues in vivo. The fact that expression of serpins A1 and $\mathrm{A} 3$, which are the main neutrophil elastase and cathepsin $\mathrm{G}$ inhibitors in blood and tissues, is repressed in the BM following administration of G-CSF or cyclophosphamide suggests that these agents not only promote neutrophil protease release but also dampens expression of the physiological inhibitors leading to HSPC mobilization [24].

Neutrophils have also been implicated in mediating apoptosis and reduction in CXCL12 production in BM osteolineage stromal cells during G-CSF mobilization, reportedly via a reactive oxygen species (ROS)-dependent mechanism which can be partially abrogated with antioxidant N-acetyl cysteine treatment [17]. As G-CSF has been shown to ramp up ROS activity in HSPCs via the c-Met/ mTOR/FOXO3 signalling axis [25], it is plausible that elevated ROS produced by neutrophils during G-CSF mobilization contributes to the general heightened ROS levels in HSPCs and their subsequent egress from the BM.

Unexpectedly however, in C57BL/6 mice lacking the genes encoding neutrophil elastase (Elane), cathepsin G (Ctsg), or dipeptidyl peptidase I (Ctsc) necessary for the functional maturation of these leukocyte serine-proteasesthat are also infused with a pan-MMP inhibitor, CFC mobilization in response to G-CSF was normal compared to wild-type C57BL/6 mice [26]. Does this mean that neutrophil proteases and neutrophil are unnecessary or not involved in HSPC mobilization? To-date we have not fully answered this question. Indeed, it must be noted firstly that the protease-deficient strains were all backcrossed into the C57BL/6 genetic background which releases very low levels of neutrophil elastase, cathepsin G and MMP-9 in response to G-CSF and CYP in the BM and have three to fivefold lower HSPC mobilization compared to BALB/c and $129 \mathrm{SvJ}$ strains. Therefore, C57BL/6 strain may not be the best inbred strain to study neutrophil protease-mediated mechanisms. Secondly, germinal deletion of these protease genes may let the organism adapt by developing redundant pathways. Indeed administration of neutrophil elastase and cathepsin $\mathrm{G}$ inhibitors in wild-type animals has been reported to reduce HSPC mobilization in response to G-CSF [23]; whereas infusion of serpin A1 or an neutralizing anti-serpinal antibody in mice, respectively inhibited or enhance HSPC mobilization in response to IL-8 [27]. Finally in experiments in C57BL/6 mice in which HSPC mobilization is induced by injections of clodronate-loaded liposomes to deplete mononucleated phagocytes such as macrophages, additional deletion of the Csf $3 r$ gene delays mobilization in response to these liposomes [28]. Therefore, neutrophil and their proteases may amplify HSPC mobilization by cleaving VCAM-1, SCF and CXCL12 in HSPC niches, while additional mechanisms are obviously in play when protease release is reduced or inhibited.

\section{Macrophages}

The fact that homozygous deletion of neutrophil protease genes did not result in reduced HSPC mobilization in C57BL/6 mice led to studies to discover alternative mechanisms. Clues about the role of BM macrophages in HSPC mobilization in response to G-CSF or CYP were the initial observations that (1) G-CSF ablates osteoblasts and bone formation in humans and mice [29-31] and (2) a specialized population of macrophages called osteomacs forms a canopy over active osteoblasts on endosteal and periosteal 
surfaces and are necessary to maintain osteoblasts and bone formation in vivo [32]. We found that G-CSF and cyclophosphamide depletes $\mathrm{F} 4 / 80^{+}$osteomacs on endosteal surfaces associated with down-regulated expression of the $S c f$, Cxcl12 and Vcaml genes [28]. G-CSF also depletes other macrophage subsets in the BM such as erythroblastic island macrophages leading to medullar anemia [33]. In two models of targeted macrophage depletion, such as injection of clodronate-loaded liposomes or macrophage-Fas-inducedapoptosis (Mafia) transgenic mice, macrophage depletions also leads to dramatic reduction of Cxcl12, Scf, Vcam1, and Angptl expression in the BM, loss of osteomacs and osteoblasts mobilization of HSPCs into the blood [28]. This effect of G-CSF may be autonomous to macrophages as macrophages also express $C s f 3 r$ [33].

This initial study was rapidly confirmed by two other groups showing firstly that $\mathrm{CD} 169^{+}$macrophages promote Nestin ${ }^{+}$MSPC secretion of CXCL12 and expression of Vcaml and $S c f$, leading to HSC retention in the niche whereas selective depletion of $\mathrm{CD} 169^{+}$macrophages leads to down-regulated expression of these genes and HSPC mobilization [34]. Secondly, Csf3r knock-out mice containing a single $C s f 3 r$ transgene driven by $C d 68$ promoter to restrict G-CSFR expression to monocytes/macrophages exhibited HSPC mobilization, loss of endosteal osteoblasts and reduced expression of $\mathrm{Cxcl12}$ despite their profound neutropenia [35]. This suggests that G-CSF signalling in and subsequent depletion of macrophages and monocytes is sufficient to initiate HSPC mobilization.

Interestingly, macrophage activation and expansion can also lead to HSPC mobilization, as suggested by recent findings showing that liver-secreted leukocyte cell-derived chemotaxin 2 (LECT2) mobilizes HSPC via macrophages and osteolineage cells expressing the LECT2 receptor CD209a via a complex mechanisms involving TNF- $\alpha$ and increased expression of CD26 (dipeptidyl peptidase IV) and cleavage and inactivation of CXCL12 [36]. These studies shed light on the complex mechanisms behind macrophage regulation of HSPC mobilization, and the identity of the precise macrophage population and its mediator cytokine(s) in regulating HSPC retention remains an area of exciting research.

\section{Osteoclasts}

Osteoclasts (OCs) are hematopoietic derived bone resorbing cells found adjacent to osteocytes and osteoblasts at the endosteum implicated in HSC niche regulation by remodelling of the $\mathrm{BM}$ cavity. Since osteoclastogenesis and bone resorption releases extracellular $\mathrm{Ca}^{2+}$ from the bone into the marrow space [37] and that disruption of calcium sensing receptor $(\mathrm{CaR})$ on HSPCs either pharmacologically or using knockout models resulted in significant extramedullary haematopoiesis [38, 39], OCs may regulate HSC retention in the endosteum by modulating the $\mathrm{Ca}^{2+}$ gradient.

Both human and mouse OC express $\mathrm{Cxcr} 4$ and $\mathrm{Cxcl12}$ [40-42]. The number of tartrate-resistant acid phosphatase positive $\left(\mathrm{TRAP}^{+}\right) \mathrm{OC}$ as well as bone resorption increase in association with G-CSF treatment [29]. Mouse receptor activator of nuclear factor- $\kappa \mathrm{B}$ ligand (RANKL)-stimulated OCs have been shown to enhance G-CSF-induced hematopoietic progenitor mobilization via CXCL12, matrix metalloproteinase-9 (MMP-9) and cathepsin K-dependent mechanisms [42]. However, one study has also shown that pharmacological inhibition of OCs using bisphosphonate led to slightly reduced repopulation capacity of HSCs [43].

However, several other studies have called the involvement of osteoclasts in HSPC mobilization into question. Macrophage depletion strategies using clodronate liposome and the macrophage-Fas-induced apoptosis (Mafia) models both reduced osteoclast numbers, yet resulted in robust HSC mobilization [28]. Administration of bisphosphonates and anti-RANKL antibody to suppress OC activity instead enhanced mobilization of HSPCs in response to G-CSF [28, 29, 44]. Furthermore, the timing of G-CSF-induced elevation in osteoclast number and activity seems to occur after the peak of HSPC mobilization [28, 29]. Studies utilising gene knockout models with defective OCs at birth are confounded by a perturbed BM niche, including osteopetrosis, leading to abnormal levels and response of HSPCs depending on the severity of the OC defect [44, 45]. A recent study by Daniel Link's group employing two mouse models with selective OC depletion without osteopetrosis again found no effect on G-CSF-induced HSPC mobilization [46]. These later studies conclusively demonstrate that $\mathrm{OC}$ are dispensable for HSPC mobilization.

\section{Mesenchymal cells}

\section{Mesenchymal stromal progenitor cell (MSPC) and $\mathrm{CXC12}$-abundant reticular (CAR) cells}

Mesenchymal stromal progenitor cells (MSPCs) are defined by their capacity to generate differentiated osteoblasts, chondrocytes and adipocytes in vitro and in vivo if implanted in the correct environment. Although present in the BM and other tissues such as the adipose, their precise location remained hampered by the lack of specific markers [47-49]. CD146 is used to identify MSPC in humans [50-52], and help demonstrate that these cells are perivascular similar to pericytes and that in addition to their ability to generate bone, cartilage and adipose when implanted in mice, MSPC also express HSC regulators such as Kit 
ligand and CXCL12 and could support HSC maintenance in vitro (reviewed in [53]).

Identification and visualisation of murine MSPCs in the $\mathrm{BM}$ has proven protracted. Initial studies using mice in which a green fluorescent protein (GFP) was knockedin the $\mathrm{Cxcl} 12$ gene, showed that primitive HSPC and HSC were adjacent to CXCL12-abundant reticular (CAR) cells that were mostly perivascular in the BM [54]. Different groups have used different genetic approaches to identify HSC niche supportive cells such as nestin-GFP transgenic mice in which GFP is driven by a nestin gene promoter and Lepr-Cre mice to identify leptin receptor expressing pericytes. Others have identified MSPCs as CD45- Ter119$\mathrm{CD} 31^{-} \mathrm{Sca}^{+}$platelet-derived growth factor receptor (PDGFR) $-\alpha^{+}$(or P $\alpha \mathrm{S}$ ) cells [55] whereas CAR cells are reported to be PDGFR $\alpha^{+} \mathrm{Scal}^{-}$[56]. Despite some controversies on which MSPC subsets-CAR cells, $\mathrm{P} \alpha \mathrm{S}$ or pericytes -express these different markers, all these cells express high levels of Cxcl12, Scf, Angptll and Vcaml mRNA [47, 56]. Importantly, administration of G-CSF decreases expression of such HSC retention factors by these BM mesenchymal cells, suggesting an essential role in HSC retention within the BM [47]. Likewise depletion of CAR cells [56], nestin-expressing cells [34, 47], or conditional deletion of the Cxcl12 gene in MSPCs [57] results in HSPC mobilization. Interestingly, depletion of macrophages leads to a similar outcome, with reduced expression of Cxcl12, Scf, Angptl and Vcaml mRNA in MSPCs [34]. Therefore, specialized macrophages may act as a relay between MSPC that do not express G-CSFR and G-CSF itself to down-regulate the expression of CXCL12, VCAM-1, and transmembrane SCF, which retain HSPC within their niche. The precise identity of macrophages regulating mesenchymal cells in HSC niches is not established and neither are the mechanisms involved understood.

\section{Osteolineage cells}

MSPCs migrate from perivascular sites towards the bone down the osteolineage differentiation pathway into osteoblasts [58], which form a layer of bone depositing cells before being entrapped in the mineralized compact bone matrix and become osteocytes. Osteoblasts were initially thought to be a candidate HSC niche component as suggested by gene deletion studies. However, the earlier mouse studies demonstrating osteoblast regulation of HSC were misinterpreted because the phenotype used to identify "HSC" was limited to $\mathrm{Lin}^{-} \mathrm{Kit}^{+} \mathrm{Sca}-1^{+}, 95 \%$ of which is made of primitive HPC, not HSC [59, 60]. Subsequent studies using more sophisticated phenotypes such as $\mathrm{Lin}^{-} \mathrm{CD} 48^{-} \mathrm{CD} 244^{-} \mathrm{CD} 150^{+}$, or transgenic and knockin mice with fluorescent reporters more restricted to HSC, combined with enhanced in vivo imaging techniques showed that only a small proportion of HSC are found in contact with osteoblasts with most HSC in a perivascular location [54, 61-65]. The higher density of HSC near the endosteum may be a reflexion of the high vascularization of the endosteal regions of the BM [62]. Furthermore, osteoblast depletion using transgenic biglycan deficient mice [66], or expansion via strontium administration [67] produced no change in HSC number or mobilization into the blood although depletion of osteoblasts in the Col2.3-HTK transgenic model resulted in HSPC mobilization 2 weeks after osteoblast depletion suggesting possible indirect effects. Furthermore, three recent studies with conditional deletion of the $C x c l 12$ or $S c f$ genes from mature osteoblasts using Ocn-Cre or the 2.3-kb fragment of the rat collagen 1a (Col2.3)-Cre did not result HSC mobilization [48, 57, 68]. However, consistent with the observation that G-CSF ablates osteoblasts and medullar B lymphopoiesis [69] (B lymphocyte progenitors need contact with osteoblasts and CXCL12 to develop [70, 71]), conditional deletion of Cxcl12 gene in osteoblasts resulted in the loss of medullar B lymphopoiesis [57, 68].

Terminally differentiated from osteoblasts and embedded in the cortical bone matrix, osteocytes have recently been implicated in HSC mobilization in response to G-CSF. In transgenic mice expressing the human diphtheria toxin receptor under the control of the Dmpl gene promoter, treatment with diphtheria toxin which kills osteocytes in these mice abolished HSPC mobilization in response to G-CSF [72]. However, the recent discovery that the 9.6-kb Dmpl promoter employed to deplete osteocytes in these mice is also expressed in approximately $30 \%$ of CAR cells throughout the BM [73] questions the validity of this interpretation as depletion of CAR cells is also known to cause HSPC mobilization [56].

The exact role of immature osteoprogenitors expressing the SP7/osterix transcription factor also remains uncertain. $\mathrm{SP}^{+}$osteoprogenitors are important to maintain normal HSPC niches as conditional deletion of the Dicerl gene essential for microRNA generation, results in myelodysplasia [74], as does conditional deletion of the $S b d s$ gene which regulates ribosome formation in $\mathrm{SP}^{+}$osteoprogenitor cells. However, the role of osteoprogenitors as genuine HSC niche cells remains controversial as conditional deletion of the $\mathrm{Scf}$ or $\mathrm{Cxcl12}$ genes in $\mathrm{SP}^{+}$osteoprogenitors did not impair HSC number and function in the BM. Nevertheless conditional deletion of the $\mathrm{Cxcl12}$ gene in $\mathrm{SP}^{+}$ osteoprogenitors did cause mobilization of CFU-C, which are mostly myeloid progenitors [57].

\section{Endothelial cells (ECs)}

EC express many of the factors necessary to HSC maintenance such as SCF, VCAM-1, CXCL12, angiopoietin-1, 
or E-selectin. Furthermore, conditional deletion of either the Scf gene or Cxcl12 gene in endothelial cells results in a dramatic loss of HSC within the BM [48, 68]. It remains, however, controversial whether EC express the G-CSF receptor and can thus directly respond to G-CSF administered during therapeutic HSC mobilization. However, endothelial cells themselves are the major source of endogenous G-CSF production in response to physiological stress or bacterial infections [75]. This is relevant to bacterial infections as lipopolysaccharide (LPS) mobilizes HSC into blood [42] and spleen [76]. Indeed, HSC mobilization in response to LPS is impaired in mice lacking the $C s f 3 r$ gene, showing that the robust HSC mobilization that takes place in response to G-CSF is indirectly mediated by endogenous release of G-CSF. Using mice floxed for tissue specific myeloid differentiation response gene Myd88 with LPS administration to model severe infection, it was shown that endothelial cells, not MSC or macrophages were the primary producers of G-CSF after inflammation [75]. In these models $M y d 88$ gene was conditionally deleted using $P D G F \beta$-Cre (MSPC cells) Nestin-Cre (MSPC and nerves), LysM-Cre (macrophages, monocytes, granulocytes), Tie2Cre (endothelial) cell populations [75]. Moreover the G-CSF production by endothelial cells during inflammation is responsible for triggering emergency granulopoiesis, and BM myeloid progenitor skewing [75], as well as HSC egress into the blood during acute inflammatory response [76].

Endothelial cells also serve as the gatekeepers at the BM-blood interface, controlling bidirectional transendothelial migration (TEM) of leukocytes to and from different organ systems, this function may be perturbed during G-CSF mobilization [77, 78]. In the BM, G-CSF-induced upregulation of sinusoidal endothelial CD26 (dipeptidyl peptidase 4) has been indicated to be important for HSPC transendothelial migration and mobilization via cleavage of full length neuropeptide Y (NPY) to NPY3-36 [79]. The in vivo effects of G-CSF on EC are also influenced by the involvement of infiltrated neutrophils and their secreted proangiogenic cytokines such as vascular endothelial growth factor (VEGF) and basic fibroblast growth factor (bFGF), which leads to neovascularisation $[80,81]$ and dissociation of tight junction element vascular endothelial cadherin (VE-cad) [82, 83]. However, it must be noted that G-CSF treatment does not lead to measurable vascular leakage as determined by Evans Blue perfusion [84]. This is in contrast with CYP treatment, which disrupts the continuity of BM vascular beds $[15,85]$ and dramatically increases vascular leakage in the BM [84]. The fact that cyclophosphamide causes vascular leakage in the BM while G-CSF does not may explain in part why cyclophosphamide is a more potent mobilizer than G-CSF, and that these two agents synergize together.
It is possible that interactions with adhesion molecules, cytokines and other membrane bound factors on ECs may alter the activation state of mobilized HSC during transmigration - an area that would benefit from further study.

The majority of HSCs are perivascular in location [54, $61,65,68]$ and although endothelial cells from both arterioles and sinusoids express key factors involved in retention of HSC -CXCL12, SCF, VCAM-1, conditional deletion of the Cxcl12 and Scf genes using Tie2-Cre mice did not trigger mobilization of HSC even though an overall reduction in BM HSC number and reconstitution potential occurred $[48,68]$ further demonstrating the complexity of this system.

Finally, part of the confusion of whether EC express G-CSF receptor may be due to the potential of residual macrophage contamination in the primary EC cultures used in many studies. Indeed macrophage populations can persist for several cell passages in such cell cultures influencing EC responses. Furthermore, endothelial lineage tracing studies in mice commonly use Tie 2 as an endothelial reporter. Tie 2 is also expressed by some macrophage subsets [86], again highlighting the uncertainty in interpretation of data from targeted Cre mouse strains.

\section{Sympathetic nervous system (SNS)}

The $\mathrm{BM}$ is richly innervated with myelinated and nonmyelinated neurons [87], with sympathetic nerve fibre endings found in close contact with bone-lining osteoblasts and osteoclasts [88], as well as perivascular $\mathrm{Nestin}^{+}$MSCs [47] and known to control homeostatic bone remodelling via $\beta$-adrenergic receptor ( $\beta$-AR) on osteoblasts $[89,90]$. In humans, chronic stress-induced $\beta$-AR signalling in the bone marrow has been suggested to reduce $\mathrm{Cxcl12}$ expression on stromal cells and promote mobilization of hematopoietic cells [91], and catecholaminergic neurotransmitters dopamine and adrenaline directly induce migration and proliferation of $\mathrm{CD} 34^{+}$HSPCs in vitro and in immunodeficient mice, with greater effect observed in vivo [92]. The first conclusive evidence of sympathetic nervous regulation of HSCs came from two consecutive studies by the Paul Frenette's group that showed photic-controlled circadian oscillations of adrenergic signals inversely regulate CXCL12 secretion in the BM, ultimately regulating release of HSPCs into the bloodstream during steady state [31, 93]. They have since reported electromechanical associations between $\mathrm{Nestin}^{+}$MSCs and noradrenergic neurons, and that $\beta$-AR agonists administration downregulated Nestin ${ }^{+}$ MSC expression of HSC retention genes Cxcl12, Vcam-1, and $S c f$ [47].

As the regulation of HSPC retention by the SNS is relatively new, knowledge regarding the effect of G-CSF acting via the sympathetic nervous system to elicit HSPC 
mobilization is scarce. Sympathetic neurons are known to express both G-CSF and G-CSFR, with G-CSF as a known neuroprotective agent [94-96]. In sympathetic neurons, G-CSF administration was reported to upregulate production of the rate limiting enzyme in noradrenaline synthesis tyrosine hydroxylase, as well as downregulate catecholamine-inactivating enzymes and noradrenaline uptake transporter activity, which clears noradrenaline from the synaptic cleft [97]. Overall, this leads to increased sympathetic tone and subsequent osteoblast depletion, $\mathrm{Cxcl12}$ downregulation, and HSPC mobilization, which can be attenuated by catecholaminergic neuron ablation with 6-hydroxydopamine [31].

Interestingly, cytotoxic chemotherapy drugs such as cisplatin and vincristine well known to cause peripheral neurotoxicity also cause dopaminergic/adrenergic neuron death in the BM [98]. Vincristine or cisplatin treatment in patients also results in impaired HSC mobilization in response to G-CSF [98]. Adjuvant treatment with neuroprotective 4-methylcatechol or glial cell-derived neurotrophic factor protected sympathetic nerves in the $\mathrm{BM}$ from vincristine or cisplatin and was shown to restore HSC mobilization in response to G-CSF [98]. Therefore, adrenergic nerves in the BM are necessary to maintain the function of MSPCs in HSC niches and promote HSC mobilization in response to G-CSF [98, 99].

The neurotransmitter neuropeptide Y (NPY) has also recently been discovered as an acute mobilizing agent that influences BM CXCL12 levels, apparently acting via osteoblastic Y1/MMP-9/CXCL12 axis [100]. Interestingly G-CSF, CXCR4 antagonist AMD3100, and 5-fluorouracil (5-FU) failed to mobilize HSPCs in NPY knockout mice, suggesting NPY as a downstream effector of these mobilizing agents [100]. Collectively, these studies demonstrate an essential role of the sympathetic nervous system in regulating HSPC retention and/or mobilization indirectly via modulation of the MSPC function in HSC niches.

\section{Erythrocytes}

G-CSF is well known to activate the complement cascade and complement fragment $\mathrm{C} 3$ and $\mathrm{C} 5$ activation, which seems to play a determinant role in HSPC mobilization. Indeed mice with homozygous deletion of the $C 3$ gene have enhanced mobilization in response to G-CSF [101], whereas mice lacking the $C 5$ gene have impaired mobilization in response to G-CSF [102]. The fact that immune deficient mice such as NOD/SCID cannot activate the complement cascade because they lack immunoglobulins may explain why they mobilize poorly in response to G-CSF [103].

Mechanistically, the role of C5 to promote HSC mobilization in response to G-CSF has been proposed to involve blood erythrocytes [104]. In this study, G-CSF was found to activate the complement cascade (including fragment C5), resulting in the formation the membrane attack complex that lyses erythrocytes in the peripheral blood, causing the release of hemoglobin and sphingosine-1 phosphate (S1P) (erythrocytes are a large reservoir of S1P). This phenomenon was C5-dependant as it was not observed in $C 5^{-1-}$ mice. As S1P is a potent chemoattractant of HSPC, the formation of this counter-gradient in the blood from hemolysis has been proposed to contribute to HSPC mobilization [104].

\section{HSCs}

Finally, HSC mobilization is not a fully HSC extrinsic mechanism in which the function of the niche is impaired with HSC flushing passively in the circulation as a result. Indeed HSC need to actively migrate out of their niche and intravasate across the $\mathrm{BM}$ endothelium. This is an active mechanism which involve in part the adhesive interaction between the tyrosine kinase receptor EPHB4 on sinusoidal endothelial cells and its transmembrane ligand ephrin B2 expressed by HSC [105, 106]. Indeed inhibition of EPHB4 kinase or antibody mediated neutralization of ephrin B2 impairs HSC mobilization in response to G-CSF or AMD3100 [105]. Remarkably, inhibition of EPHB4 or ephrin B2 did not impair HSC homing [105], further illustrating that HSC mobilization is not the mirror image of HSC homing as suggested in experiments performed in mice with conditional deletion of both Racl and Rac2 GTPase genes in hematopoietic cells, which cause a very pronounced HSC mobilization with only mild effect on HSC homing [3, 107].

\section{Conclusion}

Approximately 100,000 mobilized HSC transplants are performed worldwide each year. Therapeutic mobilization for harvesting HSC for transplant was performed decades before the research on understanding how administration of cytokines causes the egress of HSC into the blood. This research has shown that it is not a HSC intrinsic event but rather the disruption of a complex HSC: niche unit in the $\mathrm{BM}$ with the severing of HSC retention factors that leads to their mobilization into the blood (summarised in Table 1; Fig. 1), discoveries that lead to the emerging field of niche biology. This example of 'reverse translation' (bedside to bench) has now revealed many aspects and a better appreciation of the dynamics of HSC:BM niche interactions. These discoveries have led to targeted strategies to further improve therapeutic HSC transplantation protocols with the addition of new synthetic agents directly targeting HSC 
niche retention factors (such as AMD3100/Plerixafor which blocks CXCR4) to boost mobilization efficiency in combination with G-CSF. In the future, these studies are likely to provide alternative HSC mobilization regimes that do not require perturbation of the BM niche as occurs with the current regimes involving G-CSF and cyclophosphamide.

Acknowledgements JPL and IGW are supported by the Mater Foundation and Research Fellowships from the National Health and Medical Research Council (NHMRC) of Australia (\#1044091 for JPL) (\#1108352 for IGW). JT is supported by the Australian Postgraduate Award from the Australian Government Department of Education and Training. The authors' work described in this review was supported by NHMRC project Grants (JPL) 1046590, 434515, 35406 and (IGW) 1083198, 543706, 1130273; by Flack Trust, Australia grant (IGW) and Queensland Government Smart Futures Fellowship (IGW).

\section{Compliance with ethical standards}

Conflict of interest The authors have no conflicts of interest to declare.

\section{References}

1. Eidenschink L, DiZerega G, Rodgers K, Bartlett M, Wells DA, Loken MR. Basal levels of CD34 positive cells in peripheral blood differ between individuals and are stable for 18 months. Cytom B Clin Cytom. 2012;82:18-25.

2. Abkowitz JL, Robinson AE, Kale S, Long MW, Chen J. Mobilization of hematopoietic stem cells during homeostasis and after cytokine exposure. Blood. 2003;102:1249-53.

3. Levesque JP, Helwani FM, Winkler IG. The endosteal 'osteoblastic' niche and its role in hematopoietic stem cell homing and mobilization. Leukemia. 2010;24:1979-92.

4. Ratajczak MZ, Kim CH, Wojakowski W, Janowska-Wieczorek A, Kucia M, Ratajczak J. Innate immunity as orchestrator of stem cell mobilization. Leukemia. 2010;24:1667-75.

5. To LB, Levesque JP, Herbert KE. How I treat patients who mobilize hematopoietic stem cells poorly. Blood. 2011;118:4530-40.

6. Bonig H, Papayannopoulou T. Hematopoietic stem cell mobilization: updated conceptual renditions. Leukemia. 2013;27:24-31.

7. Kruger P, Saffarzadeh M, Weber ANR, Rieber N, Radsak $M$, von Bernuth $\mathrm{H}$, et al. Neutrophils: between host defence, immune modulation, and tissue injury. PLoS Pathog. 2015;11:e1004651.

8. Kolaczkowska E, Kubes P. Neutrophil recruitment and function in health and inflammation. Nat Rev Immunol. 2013;13:159-75.

9. Furze RC, Rankin SM. The role of the bone marrow in neutrophil clearance under homeostatic conditions in the mouse. FASEB J. 2008;22:3111-9.

10. Lucas M, Stuart LM, Savill J, Lacy-Hulbert A. Apoptotic cells and innate immune stimuli combine to regulate macrophage cytokine secretion. J Immunol. 2003;171:2610-5.

11. Casanova-Acebes M, Pitaval C, Weiss LA, Nombela-Arrieta $\mathrm{C}$, Chevre R, A-Gonzalez N, et al. Rhythmic modulation of the hematopoietic niche through neutrophil clearance. Cell. 2013;153:1025-35.

12. Levesque JP, Hendy J, Takamatsu Y, Williams B, Winkler IG, Simmons PJ. Mobilization by either cyclophosphamide or granulocyte colony-stimulating factor transforms the bone marrow into a highly proteolytic environment. Exp Hematol. 2002;30:440-9.

13. King AG, Horowitz D, Dillon SB, Levin R, Farese AM, MacVittie TJ, et al. Rapid mobilization of murine hematopoietic stem cells with enhanced engraftment properties and evaluation of hematopoietic progenitor cell mobilization in rhesus monkeys by a single injection of SB-251353, a specific truncated form of the human CXC chemokine GRObeta. Blood. 2001;97:1534-42.

14. Pruijt JF, Verzaal $P$, van Os R, de Kruijf EJ, van Schie ML, Mantovani A, et al. Neutrophils are indispensable for hematopoietic stem cell mobilization induced by interleukin- 8 in mice. Proc Natl Acad Sci USA. 2002;99:6228-33.

15. Szumilas P, Barcew K, Baskiewicz-Masiuk M, Wiszniewska B, Ratajczak MZ, Machalinski B. Effect of stem cell mobilization with cyclophosphamide plus granulocyte colony-stimulating factor on morphology of haematopoietic organs in mice. Cell Prolif. 2005;38:47-61.

16. Pruijt JF, Willemze R, Fibbe WE. Mechanisms underlying hematopoietic stem cell mobilization induced by the CXC chemokine interleukin-8. Curr Opin Hematol. 1999;6:152-8.

17. Singh P, Hu P, Hoggatt J, Moh A, Pelus LM. Expansion of bone marrow neutrophils following G-CSF administration in mice results in osteolineage cell apoptosis and mobilization of hematopoietic stem and progenitor cells. Leukemia. 2012;26:2375-83.

18. Liu FL, PoursineLaurent J, Link DC. The granulocyte colonystimulating factor receptor is required for the mobilization of murine hematopoietic progenitors into peripheral blood by cyclophosphamide or interleukin-8 but not flt-3 ligand. Blood. 1997;90:2522-8.

19. Winkler IG, Levesque JP. Mechanisms of hematopoietic stem cell mobilization: when innate immunity assails the cells that make blood and bone. Exp Hematol. 2006;34:996-1009.

20. Levesque JP, Takamatsu Y, Nilsson SK, Haylock DN, Simmons PJ. Vascular cell adhesion molecule-1 (CD106) is cleaved by neutrophil proteases in the bone marrow following hematopoietic progenitor cell mobilization by granulocyte colony-stimulating factor. Blood. 2001;98:1289-97.

21. Levesque JP, Hendy J, Takamatsu Y, Simmons PJ, Bendall LJ. Disruption of the CXCR4/CXCL12 chemotactic interaction during hematopoietic stem cell mobilization induced by GCSF or cyclophosphamide. J Clin Investig. 2003;111:187-96.

22. Levesque JP, Hendy J, Winkler IG, Takamatsu Y, Simmons PJ. Granulocyte colony-stimulating factor induces the release in the bone marrow of proteases that cleave c-KIT receptor (CD117) from the surface of hematopoietic progenitor cells. Exp Hematol. 2003;31:109-17.

23. Petit I, Szyper-Kravitz M, Nagler A, Lahav M, Peled A, Habler $\mathrm{L}$, et al. G-CSF induces stem cell mobilization by decreasing bone marrow SDF-1 and up-regulating CXCR4. Nat Immunol. 2002;3:687-94.

24. Winkler IG, Hendy J, Coughlin P, Horvath A, Levesque JP. Serine protease inhibitors serpina1 and serpina3 are down-regulated in bone marrow during hematopoietic progenitor mobilization. J Exp Med. 2005;201:1077-88.

25. Tesio M, Golan K, Corso S, Giordano S, Schajnovitz A, Vagima $\mathrm{Y}$, et al. Enhanced c-Met activity promotes G-CSF-induced mobilization of hematopoietic progenitor cells via ROS signaling. Blood. 2011;117:419-28.

26. Levesque JP, Liu F, Simmons PJ, Betsuyaku T, Senior RM, Pham C, et al. Characterization of hematopoietic progenitor mobilization in protease-deficient mice. Blood. 2004;104:65-72.

27. van Pel M, van Os R, Velders GA, Hagoort H, Heegaard PM, Lindley IJ, et al. Serpina1 is a potent inhibitor of IL-8-induced 
hematopoietic stem cell mobilization. Proc Natl Acad Sci USA. 2006;103:1469-74.

28. Winkler IG, Sims NA, Pettit AR, Barbier V, Nowlan B, Helwani $\mathrm{F}$, et al. Bone marrow macrophages maintain hematopoietic stem cell (HSC) niches and their depletion mobilizes HSCs. Blood. 2010;116:4815-28.

29. Takamatsu Y, Simmons PJ, Moore RJ, Morris HA, To LB, Levesque JP. Osteoclast-mediated bone resorption is stimulated during short term administration of granulocyte colony-stimulating factor but is not responsible for hemopoietic progenitor cell mobilization. Blood. 1998;92:3465-73.

30. Semerad CL, Christopher MJ, Liu F, Short B, Simmons PJ, Winkler I, et al. G-CSF potently inhibits osteoblast activity and CXCL12 mRNA expression in the bone marrow. Blood. 2005;106:3020-7.

31. Katayama Y, Battista M, Kao WM, Hidalgo A, Peired AJ, Thomas SA, et al. Signals from the sympathetic nervous system regulate hematopoietic stem cell egress from bone marrow. Cell. 2006;124:407-21.

32. Chang MK, Raggatt LJ, Alexander KA, Kuliwaba JS, Fazzalari NL, Schroder K, et al. Osteal tissue macrophages are intercalated throughout human and mouse bone lining tissues and regulate osteoblast function in vitro and in vivo. J Immunol. 2008;181:1232-44

33. Jacobsen RN, Forristal CE, Raggatt LJ, Nowlan B, Barbier V, Kaur S, et al. Mobilization with granulocyte colony-stimulating factor blocks medullar erythropoiesis by depleting $\mathrm{F} 4 / 80(+)$ VCAM1(+)CD169(+)ER-HR3(+)Ly6G(+) erythroid island macrophages in the mouse. Exp Hematol. 2014;42:547-61.

34. Chow A, Lucas D, Hidalgo A, Mendez-Ferrer S, Hashimoto D, Scheiermann C, et al. Bone marrow CD169+ macrophages promote the retention of hematopoietic stem and progenitor cells in the mesenchymal stem cell niche. J Exp Med. 2011;208:261-71.

35. Christopher MJ, Rao M, Liu F, Woloszynek JR, Link DC. Expression of the G-CSF receptor in monocytic cells is sufficient to mediate hematopoietic progenitor mobilization by G-CSF in mice. J Exp Med. 2011;208:251-60.

36. Lu XJ, Chen Q, Rong YJ, Yang GJ, Li CH, Xu NY, et al. LECT2 drives haematopoietic stem cell expansion and mobilization via regulating the macrophages and osteolineage cells. Nat Commun. 2016;7:12719.

37. Li Z, Kong K, Qi W. Osteoclast and its roles in calcium metabolism and bone development and remodeling. Biochem Biophys Res Commun. 2006;343:345-50.

38. Adams GB, Chabner KT, Alley IR, Olson DP, Szczepiorkowski ZM, Poznansky MC, et al. Stem cell engraftment at the endosteal niche is specified by the calcium-sensing receptor. Nature. 2006;439:599-603.

39. Lam BS, Cunningham C, Adams GB. Pharmacologic modulation of the calcium-sensing receptor enhances hematopoietic stem cell lodgment in the adult bone marrow. Blood. 2011;117:1167-75.

40. Grassi F, Piacentini A, Cristino S, Toneguzzi S, Cavallo C, Facchini A, et al. Human osteoclasts express different CXC chemokines depending on cell culture substrate: molecular and immunocytochemical evidence of high levels of CXCL10 and CXCL12. Histochem Cell Biol. 2003;120:391-400.

41. Wright LM, Maloney W, Yu X, Kindle L, Collin-Osdoby P, Osdoby P. Stromal cell-derived factor-1 binding to its chemokine receptor CXCR4 on precursor cells promotes the chemotactic recruitment, development and survival of human osteoclasts. Bone. 2005;36:840-53.

42. Kollet O, Dar A, Shivtiel S, Kalinkovich A, Lapid K, Sztainberg Y, et al. Osteoclasts degrade endosteal components and promote mobilization of hematopoietic progenitor cells. Nat Med. 2006;12:657-64.

43. Lymperi S, Ersek A, Ferraro F, Dazzi F, Horwood NJ. Inhibition of osteoclast function reduces hematopoietic stem cell numbers in vivo. Blood. 2011;117:1540-9.

44. Miyamoto K, Yoshida S, Kawasumi M, Hashimoto K, Kimura T, Sato Y, et al. Osteoclasts are dispensable for hematopoietic stem cell maintenance and mobilization. J Exp Med. 2011;208:2175-81.

45. Shivtiel S, Kollet O, Lapid K, Schajnovitz A, Goichberg $\mathrm{P}$, Kalinkovich A, et al. CD45 regulates retention, motility, and numbers of hematopoietic progenitors, and affects osteoclast remodeling of metaphyseal trabecules. J Exp Med. 2008;205:2381-95.

46. Rao M, Supakorndej T, Schmidt AP, Link DC. Osteoclasts are dispensable for hematopoietic progenitor mobilization by granulocyte colony-stimulating factor in mice. Exp Hematol. 2015;43:110-4.

47. Mendez-Ferrer S, Michurina TV, Ferraro F, Mazloom AR, Macarthur BD, Lira SA, et al. Mesenchymal and haematopoietic stem cells form a unique bone marrow niche. Nature. 2010;466:829-34.

48. Ding L, Saunders TL, Enikolopov G, Morrison SJ. Endothelial and perivascular cells maintain haematopoietic stem cells. Nature. 2012;481:457-62.

49. Kunisaki Y, Bruns I, Scheiermann C, Ahmed J, Pinho S, Zhang DC, et al. Arteriolar niches maintain haematopoietic stem cell quiescence. Nature. 2013;502:637-43.

50. Sacchetti B, Funari A, Michienzi S, Di Cesare S, Piersanti S, Saggio I, et al. Self-renewing osteoprogenitors in bone marrow sinusoids can organize a hematopoietic microenvironment. Cell. 2007;131:324-36.

51. Corselli M, Chin CJ, Parekh C, Sahaghian A, Wang W, Ge S, et al. Perivascular support of human hematopoietic stem/progenitor cells. Blood. 2013;121:2891-901.

52. Levesque JP. A niche in a dish: pericytes support HSC. Blood. 2013;121:2816-8.

53. Frenette PS, Pinho S, Lucas D, Scheiermann C. Mesenchymal stem cell: keystone of the hematopoietic stem cell niche and a stepping-stone for regenerative medicine. Annu Rev Immunol. 2013;31:285-316.

54. Sugiyama T, Kohara H, Noda M, Nagasawa T. Maintenance of the hematopoietic stem cell pool by CXCL12-CXCR4 chemokine signaling in bone marrow stromal cell niches. Immunity. 2006;25:977-88.

55. Morikawa S, Mabuchi Y, Kubota Y, Nagai Y, Niibe K, Hiratsu E, et al. Prospective identification, isolation, and systemic transplantation of multipotent mesenchymal stem cells in murine bone marrow. J Exp Med. 2009;206:2483-96.

56. Omatsu Y, Sugiyama T, Kohara H, Kondoh G, Fujii N, Kohno $\mathrm{K}$, et al. The essential functions of adipo-osteogenic progenitors as the hematopoietic stem and progenitor cell niche. Immunity. 2010;33:387-99.

57. Greenbaum A, Hsu YMS, Day RB, Schuettpelz LG, Christopher MJ, Borgerding JN, et al. CXCL12 in early mesenchymal progenitors is required for haematopoietic stem-cell maintenance. Nature. 2013;495:227-30.

58. Morrison SJ, Scadden DT. The bone marrow niche for haematopoietic stem cells. Nature. 2014;505:327-34.

59. Calvi LM, Adams GB, Weibrecht KW, Weber JM, Olson DP, Knight MC, et al. Osteoblastic cells regulate the haematopoietic stem cell niche. Nature. 2003;425:841-6.

60. Zhang J, Niu C, Ye L, Huang H, He X, Tong WG, et al. Identification of the haematopoietic stem cell niche and control of the niche size. Nature. 2003;425:836-41. 
61. Kiel MJ, Yilmaz OH, Iwashita T, Terhorst C, Morrison SJ. SLAM family receptors distinguish hematopoietic stem and progenitor cells and reveal endothelial niches for stem cells. Cell. 2005;121:1109-21.

62. Nombela-Arrieta C, Pivarnik G, Winkel B, Canty KJ, Harley B, Mahoney JE, et al. Quantitative imaging of haematopoietic stem and progenitor cell localization and hypoxic status in the bone marrow microenvironment. Nat Cell Biol. 2013;15:533-43.

63. Lo Celso C, Fleming HE, Wu JW, Zhao CX, Miake-Lye S, Fujisaki J, et al. Live-animal tracking of individual haematopoietic stem/progenitor cells in their niche. Nature. 2009;457:92-6.

64. Kiel MJ, Acar M, Radice GL, Morrison SJ. Hematopoietic stem cells do not depend on $\mathrm{N}$-cadherin to regulate their maintenance. Cell Stem Cell. 2009;4:170-9.

65. Acar M, Kocherlakota KS, Murphy MM, Peyer JG, Oguro $\mathrm{H}$, Inra $\mathrm{CN}$, et al. Deep imaging of bone marrow shows non-dividing stem cells are mainly perisinusoidal. Nature. 2015;526:126-30.

66. Kiel MJ, Radice GL, Morrison SJ. Lack of evidence that hematopoietic stem cells depend on N-cadherin-mediated adhesion to osteoblasts for their maintenance. Cell Stem Cell. 2007;1:204-17.

67. Lymperi S, Horwood N, Marley S, Gordon MY, Cope AP, Dazzi F. Strontium can increase some osteoblasts without increasing hematopoietic stem cells. Blood. 2008;111:1173-81.

68. Ding L, Morrison SJ. Haematopoietic stem cells and early lymphoid progenitors occupy distinct bone marrow niches. Nature. 2013;495:231-5.

69. Winkler IG, Bendall LJ, Forristal CE, Helwani F, Nowlan B, Barbier V, et al. B-lymphopoiesis is stopped by mobilizing doses of G-CSF and is rescued by overexpression of the antiapoptotic protein Bcl2. Haematologica. 2013;98:325-33.

70. Tokoyoda K, Egawa T, Sugiyama T, Choi BI, Nagasawa T. Cellular niches controlling B lymphocyte behavior within bone marrow during development. Immunity. 2004;20:707-18.

71. Zhu J, Garrett R, Jung Y, Zhang Y, Kim N, Wang J, et al. Osteoblasts support B-lymphocyte commitment and differentiation from hematopoietic stem cells. Blood. 2007;109:3706-12.

72. Asada N, Katayama Y, Sato M, Minagawa K, Wakahashi K, Kawano H, et al. Matrix-embedded osteocytes regulate mobilization of hematopoietic stem/progenitor cells. Cell Stem Cell. 2013;12:737-47.

73. Zhang J, Link DC. Targeting of mesenchymal stromal cells by Cre-recombinase transgenes commonly used to target osteoblast lineage cells. J Bone Miner Res. 2016. doi:10.1002/jbmr.2877.

74. Raaijmakers MH, Mukherjee S, Guo S, Zhang S, Kobayashi $\mathrm{T}$, Schoonmaker JA, et al. Bone progenitor dysfunction induces myelodysplasia and secondary leukaemia. Nature. 2010;464:852-7.

75. Boettcher S, Gerosa RC, Radpour R, Bauer J, Ampenberger F, Heikenwalder M, et al. Endothelial cells translate pathogen signals into G-CSF-driven emergency granulopoiesis. Blood. 2014;124:1393-403.

76. Burberry A, Zeng MY, Ding L, Wicks I, Inohara N, Morrison SJ, et al. Infection mobilizes hematopoietic stem cells through cooperative NOD-like receptor and toll-like receptor signaling. Cell Host Microbe. 2014;15:779-91.

77. Park KW, Kwon YW, Cho HJ, Shin JI, Kim YJ, Lee SE, et al. G-CSF exerts dual effects on endothelial cells-opposing actions of direct eNOS induction versus indirect CRP elevation. J Mol Cell Cardiol. 2008;45:670-8.

78. Bussolino F, Ziche M, Wang JM, Alessi D, Morbidelli L, Cremona $\mathrm{O}$, et al. In vitro and in vivo activation of endothelial cells by colony-stimulating factors. J Clin Investig. 1991;87:986-95.

79. Singh P, Hoggatt J, Hu PR, Pelus LM. Sinusoidal endothelial CD26 cleavage of neuropeptide $\mathrm{Y}$ regulates transendothelial migration and mobilization of hematopoietic stem and progenitor cells in response to G-CSF. Blood. 2012;120:2347.

80. Ohki Y, Heissig B, Sato Y, Akiyama H, Zhu Z, Hicklin DJ, et al. Granulocyte colony-stimulating factor promotes neovascularization by releasing vascular endothelial growth factor from neutrophils. FASEB J. 2005; 19:2005-7.

81. Ai S, Cheng XW, Inoue A, Nakamura K, Okumura K, Iguchi A, et al. Angiogenic activity of bFGF and VEGF suppressed by proteolytic cleavage by neutrophil elastase. Biochem Biophys Res Commun. 2007;364:395-401.

82. Nottebaum AF, Cagna G, Winderlich M, Gamp AC, Linnepe R, Polaschegg C, et al. VE-PTP maintains the endothelial barrier via plakoglobin and becomes dissociated from VE-cadherin by leukocytes and by VEGF. J Exp Med. 2008;205:2929-45.

83. Broermann A, Winderlich M, Block H, Frye M, Rossaint J, Zarbock A, et al. Dissociation of VE-PTP from VEcadherin is required for leukocyte extravasation and for VEGF-induced vascular permeability in vivo. J Exp Med. 2011;208:2393-401.

84. Winkler IG, Barbier V, Wadley R, Zannettino AC, Williams S, Levesque JP. Positioning of bone marrow hematopoietic and stromal cells relative to blood flow in vivo: serially reconstituting hematopoietic stem cells reside in distinct nonperfused niches. Blood. 2010;116:375-85.

85. Lapidot T, Dar A, Kollet O. How do stem cells find their way home? Blood. 2005;106:1901-10.

86. Venneri MA, De Palma M, Ponzoni M, Pucci F, Scielzo C, Zonari E, et al. Identification of proangiogenic TIE2-expressing monocytes (TEMs) in human peripheral blood and cancer. Blood. 2007;109:5276-85.

87. Yamazaki K, Allen TD. Ultrastructural and morphometric alterations in bone marrow stromal tissue after 7 Gy irradiation. Blood Cells. 1991;17:527-49.

88. Mignini F, Streccioni V, Amenta F. Autonomic innervation of immune organs and neuroimmune modulation. Auton Autacoid Pharmacol. 2003;23:1-25.

89. Kondo H, Nifuji A, Takeda S, Ezura Y, Rittling SR, Denhardt DT, et al. Unloading induces osteoblastic cell suppression and osteoclastic cell activation to lead to bone loss via sympathetic nervous system. J Biol Chem. 2005;280:30192-200.

90. Togari A, Arai M, Kondo A. The role of the sympathetic nervous system in controlling bone metabolism. Expert Opin Ther Targets. 2005;9:931-40.

91. Heidt T, Sager HB, Courties G, Dutta P, Iwamoto Y, Zaltsman A, et al. Chronic variable stress activates hematopoietic stem cells. Nat Med. 2014;20:754-8.

92. Spiegel A, Shivtiel S, Kalinkovich A, Ludin A, Netzer N, Goichberg P, et al. Catecholaminergic neurotransmitters regulate migration and repopulation of immature human CD34+ cells through Wnt signaling. Nat Immunol. 2007;8:1123-31.

93. Mendez-Ferrer S, Lucas D, Battista M, Frenette PS. Haematopoietic stem cell release is regulated by circadian oscillations. Nature. 2008;452:442-7.

94. Huang HY, Lin SZ, Kuo JS, Chen WF, Wang MJ. G-CSF protects dopaminergic neurons from 6-OHDA-induced toxicity via the ERK pathway. Neurobiol Aging. 2007;28:1258-69.

95. Henriques A, Pitzer C, Dupuis L, Schneider A. G-CSF protects motoneurons against axotomy-induced apoptotic death in neonatal mice. BMC Neurosci. 2010;11:25.

96. Schneider A, Kruger C, Steigleder T, Weber D, Pitzer C, Laage $\mathrm{R}$, et al. The hematopoietic factor G-CSF is a neuronal ligand that counteracts programmed cell death and drives neurogenesis. J Clin Investig. 2005;115:2083-98.

97. Battista M, Mendez-Ferrer S, Frenette PS. Granulocyte colonystimulating factor (G-CSF) stimulates sympathetic nervous system activity in the bone marrow. Blood. 2008;112:843. 
98. Lucas D, Scheiermann C, Chow A, Kunisaki Y, Bruns I, Barrick $\mathrm{C}$, et al. Chemotherapy-induced bone marrow nerve injury impairs hematopoietic regeneration. Nat Med. 2013;19:695-703.

99. Levesque JP, Winkler IG. It takes nerves to recover from chemotherapy. Nat Med. 2013;19:669-71.

100. Park MH, Lee JK, Kim N, Min WK, Lee JE, Kim KT, et al. Neuropeptide $\mathrm{Y}$ induces hematopoietic stem/progenitor cell mobilization by regulating matrix metalloproteinase-9 activity through Y1 receptor in osteoblasts. Stem Cells. 2016;34:2145-56.

101. Ratajczak J, Reca R, Kucia M, Majka M, Allendorf DJ, Baran JT, et al. Mobilization studies in mice deficient in either C3 or $\mathrm{C} 3 \mathrm{a}$ receptor $(\mathrm{C} 3 \mathrm{aR})$ reveal a novel role for complement in retention of hematopoietic stem/progenitor cells in bone marrow. Blood. 2004;103:2071-8.

102. Lee HM, Wu W, Wysoczynski M, Liu R, Zuba-Surma EK, Kucia M, et al. Impaired mobilization of hematopoietic stem/ progenitor cells in C5-deficient mice supports the pivotal involvement of innate immunity in this process and reveals novel promobilization effects of granulocytes. Leukemia. 2009;23:2052-62.

103. Reca R, Cramer D, Yan J, Laughlin MJ, Janowska-Wieczorek A, Ratajczak J, et al. A novel role of complement in mobilization: immunodeficient mice are poor granulocyte-colony stimulating factor mobilizers because they lack complement-activating immunoglobulins. Stem Cells. 2007;25:3093-100.

104. Ratajczak MZ, Lee H, Wysoczynski M, Wan W, Marlicz W, Laughlin MJ, et al. Novel insight into stem cell mobilizationplasma sphingosine-1-phosphate is a major chemoattractant that directs the egress of hematopoietic stem progenitor cells from the bone marrow and its level in peripheral blood increases during mobilization due to activation of complement cascade/ membrane attack complex. Leukemia. 2010;24:976-85.

105. Kwak H, Salvucci O, Weigert R, Martinez-Torrecuadrada JL, Henkemeyer M, Poulos MG, et al. Sinusoidal ephrin receptor EPHB4 controls hematopoietic progenitor cell mobilization from bone marrow. J Clin Investig. 2016. doi:10.1172/ JCI87848.

106. Levesque JP, Winkler IG. Cell adhesion molecules in normal and malignant hematopoiesis: from bench to bedside. Curr Stem Cell Rep. 2016;2:356-67.

107. Cancelas JA, Lee AW, Prabhakar R, Stringer KF, Zheng Y, Williams DA. Rac GTPases differentially integrate signals regulating hematopoietic stem cell localization. Nat Med. 2005;11:886-91. 